\title{
Direct Numerical Simulation for Three-Dimensional Gas-Solid Two-Phase Jet Using Two-Way Method and Experimental Verification*
}

\author{
Shinichi YUU**, Toshihiko UMEKAGE** \\ and Masaharu TABUCHI***
}

\begin{abstract}
Current progress in the field of supercomputers has made possible the direct numerical simulation of the gas-solid two-phase jet based on the Navier-Stokes equation and the Lagrangian equation of particle motion without the use of any assumptions or simplified models. In this study, three-dimensional Eulerian air velocities and Lagrangian particle trajectories are simultaneously calculated to describe the interaction between particles and air using a two-way method. Although the mesh size is roughly seven times the Kolmogorov microscale, the calculated turbulent characteristics of air and particles (mean velocity distributions and turbulent intensity distributions) are in fairly good agreement with experimental data obtained using laser Doppler anemometry. This means that the simulation well describes the motion of large-scale eddies which play an important role in the formation of turbulent gas-solid two-phase flow.
\end{abstract}

Key Words : Computational Fluid Dynamics, Multiphase Flow, Jet, Turbulent Flow, L. D. A.

\section{Introduction}

Recent progress of computer capability has allowed researchers to approximately determine the structures of complex flow fields including turbulence, by direct numerical simulation (D. N. S.). D. N. S., including both Eulerian air velocity and Lagrangian particle trajectory calculation, permits the prediction of particle turbulent characteristics in gas-particle flows without any modeling. Since D. N. S. gives the whole instantaneous data for gas and particle motion in flow fields, the stochastic procedures of the data reveal the detailed characteristics of gas and particle flows.

When the particle mass mixture ratio $m$ is small enough, that is, $m=W / Q \rho<0.2$ in jet flows, where $Q$,

* Received 9th February, 1995. Japanese original: Trans. Jpn. Soc. Mech. Eng., Vol.60, No. 572, B (1994), pp. 1152 1160. (Received 11th August, 1993)

** Department of Mechanical Engineering, Kyushu Institute of Technology, 11 Sensuicho, Tobata ku, Kitakyushu 804, Japan

*** Fuji Photo Film Co., Ltd., 22630 Nishiazabu, Minato ku, Tokyo 106, Japan
$W$ and $\rho$ are volume flow rate of air, mass flow rate of particles and air density, the presence of particles does not affect the fluid flow structures. Therefore, the one- way method is applicable to the dilute gassolid two-phase jet ${ }^{(1)}$. However, the effect of particle existence on the air flows gradually increases as the particle mass mixture ratio increases beyond 0.2 . In this study, we simultaneously calculated three-dimensional Eulerian air velocity and Lagrangian particle trajectories, considering the mutual interactions between air and particles (two-way method) by D. N.S.

It is obvious that the best numerical scheme for simulating turbulent flow is direct numerical simulation. However, it must be realized that currently available computational storage and speed are still insufficient to describe a high Reynolds number real turbulent flow, since huge computational domains are necessary. Namely, the computational cell size is considerably larger than the Kolmogorov micro scale for high Reynolds number real turbulent flows. In this simulation, the cell size is about seven times that of the Kolmogorov microscale. Therefore, verification of the calculated results is necessary. We compared 
them with experimental data which were obtained using the laser Doppler anemometer (L. D. A.) for the gas and particle velocities and the isokinetic sampling method for the particle concentrations. We found fairly good agreement, indicating that the simulation well describes the motions of large ${ }^{-}$and mediumscale eddies which play an important role in the formation of turbulent gas-particle two-phase flow.

\section{Direct Numerical Simulation Procedure for Gas-Solid Two-Phase Jet}

Particle mass loading is large enough to have an effect on the fluid turbulence, while, at the same time, the volume fraction of the particles is small enough that particle collisions are negligible. The equation of continuity for the air phase is not changed by the presence of particles since the particle volume fraction is negligibly small. The governing equations for the air phase are the three-dimensional NavierStokes equations for incompressible fluid with the particle source term and the fluid continuity equation.

$$
\begin{aligned}
& \frac{\partial \underline{u}}{\partial t}+(\underline{u} \cdot \nabla) \underline{u}=-\nabla P+\frac{1}{R e} \nabla^{2} \underline{u}-S p \\
& \nabla \cdot \underline{u}=D D=0
\end{aligned}
$$

$\nabla, P, t, \underline{u}$ and $R e$ in Eqs. (1) and (2) are the nabra operator, static pressure, time, air velocity vector and Reynolds number $\left(=D U_{0} / \nu\right.$, where $D$ is nozzle diameter, $U_{0}$ is air velocity at the nozzle outlet and $\nu$ is kinetic viscosity), respectively, and are all nondimensional variables. The last term on the right-hand side of Eq. (1) is the particle source term which indicates the mutual interaction between particles and air flows. When the Navier-Stokes equations and the equations of particle motion are solved simultaneously, the air and the particle motion are linked by the particle source term $S p$. Substituting the drag coefficient into the equation derived by Squires and Eaton ${ }^{(2)}$ gives the equation for $S p$. We used Schiller and Naumann's ${ }^{(3)}$ experimental drag coefficient, which is applicable to flow whose particle Reynolds number is less than 800 .

$$
S p=\frac{3 \pi \mu D_{p}\left(1+0.15 R e_{p}^{0.687}\right) D n}{U_{0} \rho}\left(\underline{u}-\underline{u}_{p}\right)
$$

Here $D_{p}, n, R e, \underline{u}_{p}, \rho$ and $\mu$ are particle diameter, number of particles per unit volume, particle Reynolds number, particle velocity vector, air density and viscosity, respectively. The particle Reynolds number in Eq. ( 3 ) is unknown at this point in the calculation, so we used the Reynolds number for the previous one time step since the time step is very small (nondimensional time step $\Delta t=0.03$ corresponds to $\left.1.6 \times 10^{-6} \mathrm{~s}\right)$.

The Poisson equation for pressure $P$, Eq. (4), which can be obtained by taking the divergence of Eq. (1), is solved by the relaxation method ${ }^{(4),(5)}$.

$$
\nabla^{2} P=-[\nabla \cdot(\underline{u} \cdot \nabla) \underline{u}]-\frac{\partial D D}{\partial t}+\frac{1}{R e} \nabla^{2} D D-\nabla \cdot S p
$$

When we solve Eq. (4) numerically, the condition of continuity, $D D=0$, is not always satisfied because of various computational errors. The kinetic energy due to the convection term in Eq. (1) cannot be conserved since $D D$ is not zero. Therefore, when we use the above-mentioned procedure, numerical errors slowly accumulate and the solution finally loses its reliability. Then we used the kinetic energy conservative form, Eq. ( 5 ), for the convection term following the method of Piacsek and Williams ${ }^{(6)}$. We used the third ${ }^{-}$order upstream difference scheme for the convection terms and the second-order central difference scheme for other differential terms.

Since the density ratio of the air and particles $\rho / \rho_{p}$ in this study is equal to $5 \times 10^{-4}$, the Lagrangian equations of particle motion are simplified to the following forms :

$$
\begin{aligned}
& \frac{S t}{2\left(1+0.15 \operatorname{Re}_{p}^{0.687}\right)} \frac{d^{2} x_{p}}{d t^{2}}+\frac{d x_{p}}{d t}-u \\
& -\frac{G}{1+0.15 \operatorname{Rep}_{p}^{0.687}}=0 \\
& \frac{S t}{2\left(1+0.15 \operatorname{Re}_{p}^{0.687}\right)} \frac{d^{2} y_{p}}{d t^{2}}+\frac{d y_{p}}{d t}-v=0 \\
& \frac{S t}{2\left(1+0.15 \operatorname{Re}_{p}^{0.687}\right)} \frac{d^{2} z_{p}}{d t^{2}}+\frac{d z_{p}}{d t}-w=0,
\end{aligned}
$$

where $x_{p}, y_{p}$ and $z_{p}$ give the nondimensional particle position, $u, v$ and $w$ are gas velocity components, $G$ is the nondimensional settling velocity of a particle $(G=$ $\left.\rho_{p} D_{p}^{2} g / 18 \mu U_{0}\right)$, and $S t$ is the Stokes number ( $S t=$ $\left.\rho_{p} D_{p}^{2} U_{0} / 9 \mu D\right)$. Equations ( 5$),(6)$ and $(7)$ can be solved analytically for each computational cell since the air velocity in each computational cell is constant. Equations ( 8) and (9) show examples of their analytical solutions. $u_{p \mid t m}$ and $x_{p \mid t m}$ are the particle velocity component and the particle position in the $x$ direction, respectively, after $m$ time steps. Equations ( 8 ) and (9) contain $u_{p t m}$ and $x_{p \mid t m}$ in both right- and left-hand sides, so we obtained solutions by iterative calculation.

$$
\begin{aligned}
& u_{p \mid t m}=\frac{\left|u_{p \mid t m}-u_{-G}-G /\left(1+0.15 \operatorname{Rep}_{p}^{0.68 \bar{T}}\right)\right|}{u_{p \mid t m}-u-G /\left(1+0.15 R e_{p}^{0.68 \bar{\tau}}\right)} \mid u_{p \mid t m-1} \\
& -u-G /\left(1+0.15 \operatorname{Re}_{p}{ }^{0.687}\right) \\
& \times \exp \left\{-\frac{2\left(1+0.15 R e_{p}^{0.68 i}\right)}{S t} \Delta t\right\}+u \\
& +\frac{G}{1+0.15 \operatorname{Rep}_{p}^{0.6 \bar{\gamma}}} \\
& u_{p \mid t m}=u_{p \mid t m-1}+\frac{S t}{2\left(1+0.15 R e^{0.687}\right)} \\
& \times \frac{\left|u_{p \mid t m}-u-G /\left(1+0.15 \operatorname{Re}_{p}{ }^{0.687}\right)\right|}{u_{p \mid t m}-u-G /\left(1+0.15 \operatorname{Rep}_{p}^{0.687}\right)} \\
& \times\left|u_{p \mid t m-1}-u-G /\left(1+0.15 R e_{p}^{0.68 i}\right)\right| \\
& \times\left[1-\exp \left\{-\frac{2\left(1+0.15 R e_{p}^{0.687}\right)}{S t} \Delta t\right\}\right]
\end{aligned}
$$




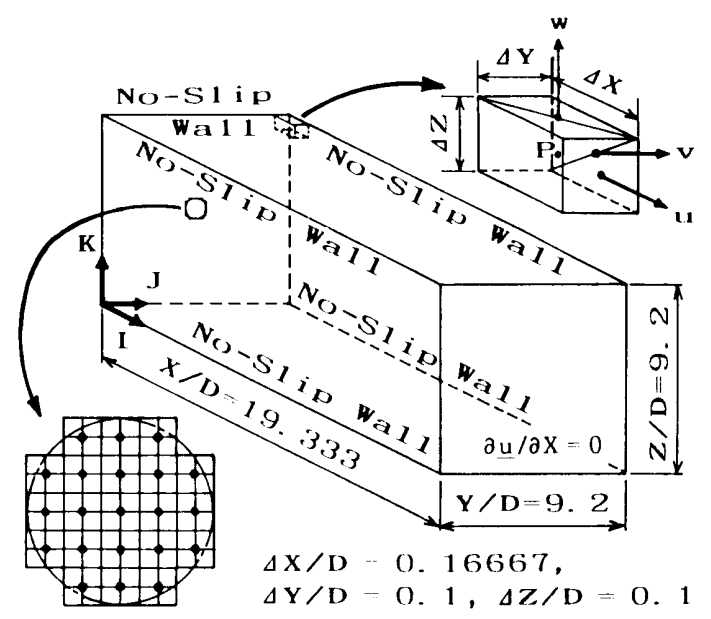

Fig. 1 Computational domain and nozzle shape

$$
\left(u+\frac{G}{1+0.15 R e_{p}^{0.687}}\right) \Delta t
$$

The Lagrangian particle trajectories consist of chains of these solution.

Figure 1 shows the computational domain and its coordinates. A round nozzle was used for the experiments, so one might expect that cylindrical coordinates would be most suitable for describing a round nozzle jet. However, when the high-order finite difference scheme is applied to a cylindrical coordi nate system, the computational errors accumulate on the jet centerline (the $x$ axis of the cylindrical coordinates) so quickly that the computation of the unsteady Navier-Stokes equation for a prolonged period cannot be completed. Therefore, we applied rectangular coordinates to the computational flow field. The round nozzle was approximated by a polygon, as shown in Fig. 1. The nondimensional cell size, $\Delta X / D$ $=0.1250$ and $\Delta Y / D=\Delta Z / D=0.0625$, and the nondimensional calculation time step, $\Delta t=0.03$, are the same in all places of the domain. The number of cells is $154 \times$ $124 \times 124$. Staggered grids shown in Fig. 1 were used. The Reynolds number is 7940 . Estimating averaged turbulence intensity to be about $5 \%$ of the nozzle outlet velocity $U_{0}$ yields the averaged Kolmogorov microscale of one-seventh of $\Delta x$. Thus eddies, of which the sizes are less than seven times the Kolmogorov microscale, are neglected in the simulation.

We used no-slip rigid wall conditions for all boundary surfaces except the nozzle outlet and the outflow boundary surface, similarly to Harlow and Welch $^{(5)}$. The effects of the side walls on the jet flow are negligible since the smallest relative distance $\eta=$ $Y / X$ between the jet centerline and the side rigid walls is 0.23 at the outflow boundary. We applied boundary conditions of $\partial u / \partial x=\partial v / \partial y=\partial w / \partial z=\partial P / \partial x=0$ to the outflow boundary. Even in this case, disturbances due to the use of inadequate outflow boundary conditions spread to the upstream portion of the flow field after the forefront of the jet reached the outflow boundary and the solution thus gradually lost its reliability. Therefore, we used the calculated results only for that portion before the forefront of the jet reaches the outflow boundary, that is, we finished our calculation at time step 3200 . It is reasonable to assume that inflow boundary conditions at the nozzle outlet were $u$ $=1.0$ and $v=w=0$ and that there were no initial disturbances. The initial particle velocity components were $u_{p 0}=0.46, v_{p 0}=0$ and $w_{p 0}=0$. The value of $u_{p 0}$ was determined by a L. D. A. experiment at the nozzle outlet. Twenty-one particles started from the 21 black points at the nozzle outlet, as shown in Fig. 1, at each time step from time step 201 to time step 3200 . The total number of calculated trajectories was 63000 $(=21 \times 3000) . \quad$ In this simulation $21 \times 8=168$ mass weighted particles, which started during 8 time steps, represent the size distribution of the feed particles (Toshiba glass beads, the number-based mean diameter $=58 \mu \mathrm{m}$, the minimum and maximum particle diameters = about $40 \mu \mathrm{m}$ and $80 \mu \mathrm{m}$ ), and the particle mass mixture ratio was $m=0.6$ at the nozzle outlet.

The velocities of air and particles at a fixed point were obtained by the interpolation method. Turbulence characteristics of gas and particles were obtained by time-averaging the instantaneous air velocities and ensemble-averaging the instantaneous particle velocities, respectively. The average particle concentration was obtained by counting the particle numbers and sizes passing through a test cell set in a cross- $^{-}$sectional area of the jet. The sizes of the test cells which are larger downstream are about $1.2-4.0$ times the computational cell size. We counted about 1600 particles in the test cell on the jet centerline at $X / D=18$ near the outflow boundary. On the other hand, the calculated particle concentrations in the outer region from the jet centerline scatter, since the number of particles counted is not very large. Thus, the calculated results in which the numbers of parti. cles counted were less than 100 were not used. Equation (10) gives the average particle concentration in the jet.

$$
C=\left(\sum_{i=1}^{N} n_{i} m_{p i}\right) /\left(t \Delta s U_{p}\right)
$$

Here $m_{p_{i}}, n_{i}, t, U_{p}$ and $U_{s}$ are particle mass and number of the $i$ th-size section passing through the test cell during the sampling time, sampling time, average particle velocity and the cross-sectional area of the test cell, respectively. The number of time steps to obtain time-averaged quantities was 1600 , from time step 1600 to time step 3200 , for $2 \leqq X / D \leqq$ 10 , and 1300 , from time step 1900 to time step 3200 , 
for $12 \leqq X / D \leqq 18$. When $D=8 \mathrm{~mm}$ and $U_{0}=14.9 \mathrm{~m} / \mathrm{s}$, 1300 time steps corresponds to $0.021 \mathrm{~s}$ in real time.

The number of eddies should be large enough to obtain an average of the independent realizations of the random dispersion process. It can be shown from the central limit theorem that the relative error in calculating the average varies as $n^{-1 / 2}$, where $n$ is the number of independent realizations. The integral time scale $T_{l}$ is the lifetime of a mean-sized eddy. Thus, the mean number of independent realizations is obtained by dividing the averaging time by $T_{l}$. Laurence's experimental integral time scale $\mathrm{e}^{(7)}$ and the minimum averaging time in this study give the minimum mean number of independent realizations $n=52$. The maximum relative error of the turbulence statistical values in obtaining the average in this simulation, therefore, is about $13 \%$.

The supercomputer we used was the FACOM VP200 , and CPU time was about 26 hours.

\section{Experimental Arrangement and Procedures}

Figure 2 shows the experimental apparatus. The solid particles were glass beads whose median diameter and density were $58 \mu \mathrm{m}$ and $2.5 \times 10^{3} \mathrm{~kg} / \mathrm{m}^{3}$. Air was supplied from the compressor. The gas velocity of the two-phase jet at the nozzle outlet was $14.9 \mathrm{~m} / \mathrm{s}$ which was set by regulating the air pressure from the compressor. The Reynolds number based on the nozzle outlet was 7940 . The solid particles supplied from an auto-feeder were led to the injector. The feed rate of particles was $5.4 \times 10^{-4} \mathrm{~kg} / \mathrm{s}$ which corresponded to the mass mixture ratio of particles and air, $m=0.6$. The air and particles were uniformly mixed in the perpendicular pipe of which the length was about $1500 \mathrm{~mm}$ from the injector to the nozzle outlet. The air and particle mixture was exhausted perpendicularly downward in the transparent rectangular

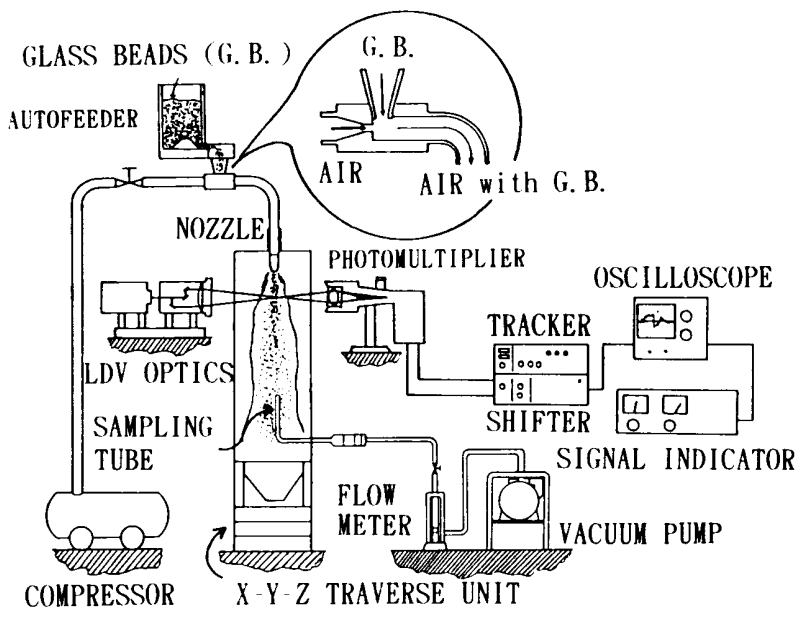

Fig. 2 Experimental setup acrylic vessel $(295 \times 295 \times 1000 \mathrm{~mm})$ from the round nozzle $(D=8 \mathrm{~mm})$ as a gas ${ }^{-}$solid two-phase jet. The jet can be traversed with the vessel for about $700 \mathrm{~mm}$ in the perpendicular direction $(X / D)$ and about 300 $\mathrm{mm}$ in the horizontal directions $(Y / D$ and $Z / D)$. This makes it possible to set a probe at any point in the jet. We measured particle velocities using a DANTEC 55 L. D. A. The tracer particles $\left(\mathrm{NH}_{4} \mathrm{Cl}\right.$ particles, median diameter $=0.8 \mu \mathrm{m}$ ) used for measuring the gas exhaust velocity $U_{0}$ of the two-phase jet were supplied with glass beads. We identified the signals of $\mathrm{NH}_{4} \mathrm{Cl}$ particles at the nozzle outlet by discriminating between signals, which represent velocities, on the monitor since the air exhaust velocity is much higher than the particle velocity at the nozzle outlet of this apparatus. The sum of the errors in measuring velocities using L. D. A. and in the averaging process of the data was about $4 \%$. The average particle concentrations were calculated from data measured by $60 \mathrm{~s}$ isokinetic sampling. The inner diameter of the sampling tube was $3.5 \mathrm{~mm}$. We examined the accuracy of the data by measuring the uniform particle concentration at the nozzle outlet. The examination showed that the error in this measuring system is less than about $7 \%$.

\section{Calculated Results of Instantaneous Velocity Vector Diagrams}

Figures 3 and 4 show the calculated instantaneous

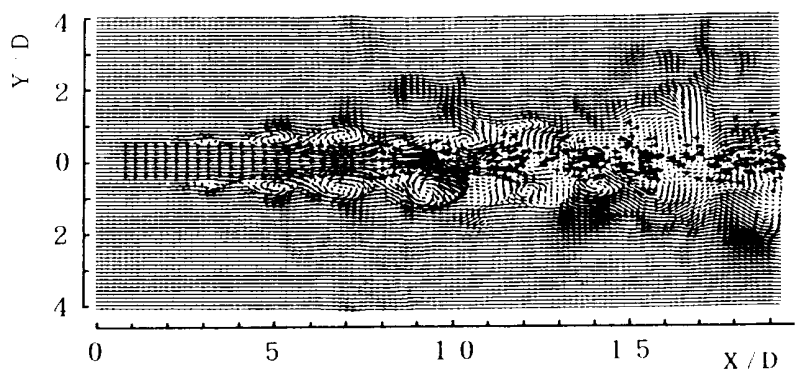

Fig. 3 Calculated instantaneous air and particle velocity vector diagram of $X Y$ plane in a gas particle two phase jet

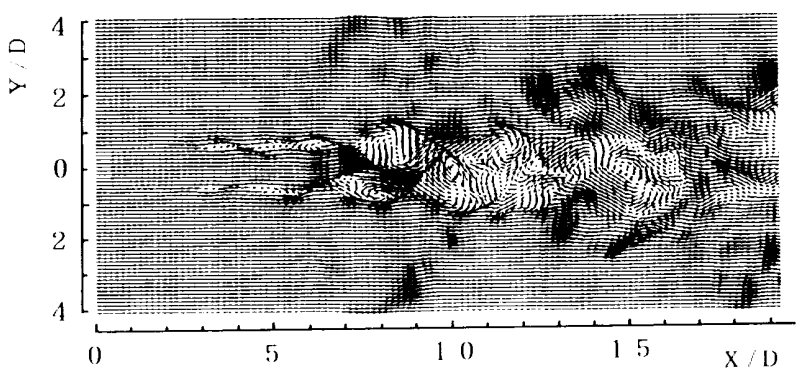

Fig. 4 Calculated instantaneous air velocity vector diagram of $X-Y$ plane in a clean air jet 
air velocity vector diagrams of the $(X / D, Y / D)$-plane $(Z / D=0)$ in a gas ${ }^{-}$particle two-phase jet and a cleanair jet, respectively, after 2500 time steps.

In drawing these diagrams, we subtracted the structure convection velocity, which we assume to be the averaged axial mean velocity at $X / D=3.5, Y / D=$ $0.5, Z / D=0$, from the instantaneous axial velocities at all cell points in the computational region and plotted them as arrows denoting their directions and scalars in order to visualize the vortices in the jet. Bold and thin line arrows indicate instantaneous particle and air velocity vectors, respectively. The subtracted velocity field is a view from a frame of reference which is moving downstream at the structure convection velocity.

Comparison of Figs. 3 and 4 shows that the largescale vortices originating at the nozzle wall start to lose their axisymmetric behavior at $X / D \fallingdotseq 6$ in the air jet ; on the other hand, the axisymmetric large-scale vortices appear until $X / D \fallingdotseq 8$ in the two-phase jet. The additional dissipation due to the presence of particles reduces the turbulent velocities of air and delays the turbulence development of a gas-particle two-phase jet as it moves downstream. Also, the presence of particles reduces the turbulent momentum transport of gas and makes the jet width narrower in the two- ${ }^{-}$phase jet.

Calculated results of instantaneous particle velocity vector diagram in Fig. 3 show that particles, whose velocity and concentration are uniform at the nozzle outlet, become clustered downstream of the jet by interacting with the large-scale vortex motions. As the stream develops into turbulence, particles are gradually transported to the outer region of the jet. Particles in the two-phase jet dodge the fluid vortices, then instantaneous low-particle-concentration regions are formed in the high-vorticity stream. The simulation by Squires and Eaton ${ }^{(8)}$ shows that the particles collect preferentially in regions of low vorticity and high strain rate in isotropic turbulent flow. Figure 3 shows that there is a similar tendency of particle motion in the two-phase jet. Some particles penetrate into large vortices downstream, since the fluid vorticity and strain rate decrease downstream in the jet. Figure 3 shows that the particles are less dispersed than in air due to their inertia and are mostly concentrated in the jet centerline region. However, the particles which become entrained in large-scale vortices are carried away from the jet centerline region by the outwardly moving flow. There may be a possibility that particles at some values of Stokes number are dispersed faster than the air, since they become entrained in large vortices and persist in motion for a longer time than the air due to their inertia ${ }^{(9)}$.

\section{Comparison of Calculated and Experimental Turbulent Characteristics}

Figure 5 shows the calculated time-averaged air and mean particle velocity distributions on the jet centerline. $U_{m}$ and $U_{p m}$ are time-averaged air and particle velocities on the jet centerline, respectively. Also, the calculated and the experimental results ${ }^{(10)}$ of the clean-air jet are shown in Fig. 5. The calculated results of the clean-air jet are in reasonably good agreement with the experimental results ${ }^{(10)}$. The calculated clean-air jet turbulence characteristics shown in Figs. 6, 7 and 8, as mentioned later, are also in reasonably good agreement with experimental results $^{(11),(12)}$ of the clean-air jet. This agreement shows the reliability of D.N.S. using the finite difference scheme in the present investigation.

In the upstream region $X / D \leqq 10$, particles are accelerated by air whose velocity is considerably higher than the particle velocity; for example, the particle velocity is 0.43 times the air velocity at the nozzle outlet. Since the air velocity in the jet decreases downstream, the particle velocity becomes equal to the air velocity at $X / D \fallingdotseq 10$. Thereafter, the particles are decelerated by air whose velocity is lower than the particle velocity, since the decay of particle velocity is slower due to the large inertia. There is a slight difference between calculated air velocities on the jet centerlines of the two-phase and clean air jets. In the upstream region $X / D \leqq 10$, the air velocities of the two-phase jet are slightly lower than those of the clean-air jet, since the air is decelerated by lower-speed particles. On the other hand, air velocities of the two-phase jet in the downstream region $X / D \geqq 10$ are slightly higher than those of the clean-air jet since particles, whose velocities become higher than that of air, due to their individual inertias, draw the air into the two-phase jet. However, the

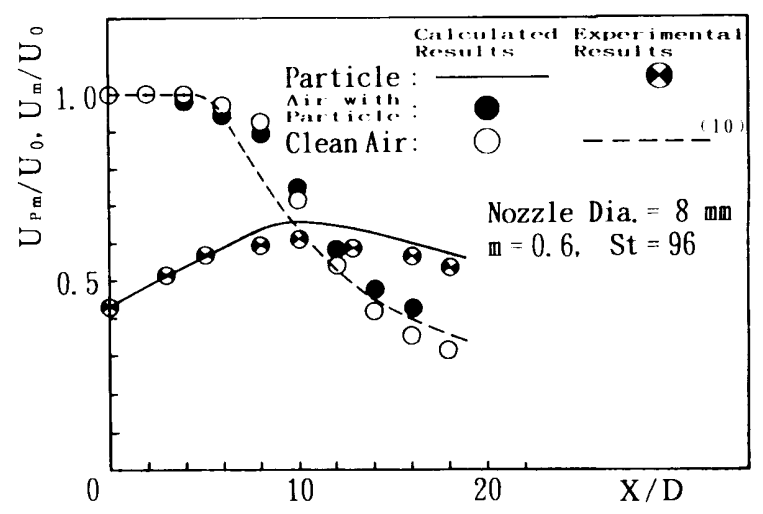

Fig. 5 Time averaged air and mean particle velocity distributions on the jet centerline 


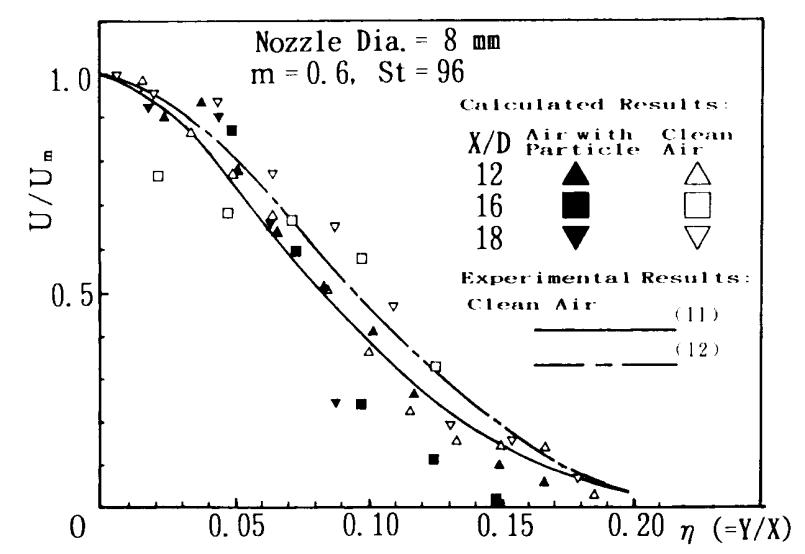

Fig. 6 Time averaged air axial velocity distributions

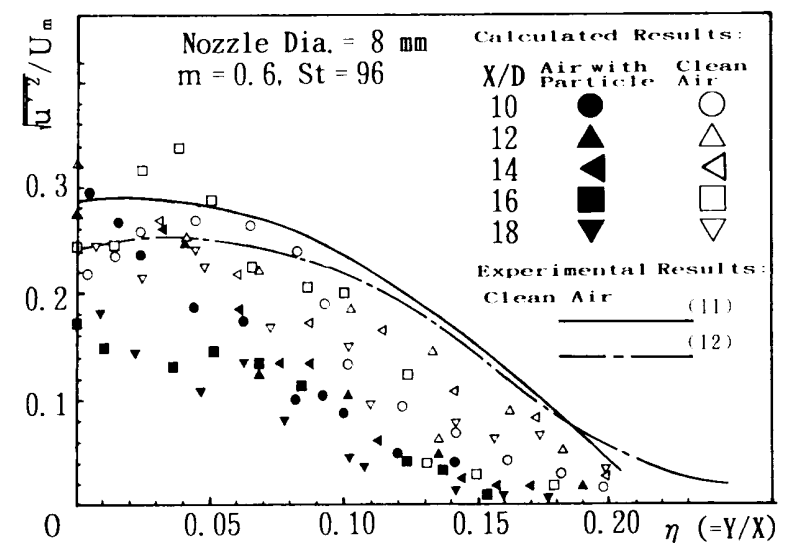

Fig. 7 Air axial turbulent intensity distributions

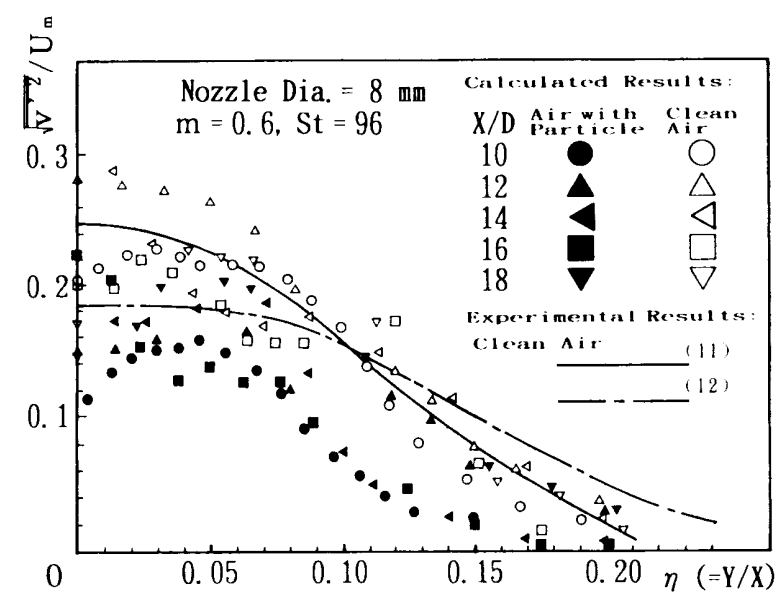

Fig. 8 Air radial turbulent intensity distributions

mass mixture ratio $m=0.6$ of this study is not so large that the mean motions of the jet would be greatly changed.

Figure 6 shows the radial distributions of the time averaged air axial velocity in the jets. The calculated results for the clean-air jet represent the clean air experimental data ${ }^{(11), 12)}$. The calculated results for the clean-air jet show that similarity in the velocity distributions is attained in the far region from the nozzle outlet. The spread of the two-phase jet is slightly narrower than that of the clean-air jet. This means that the presence of particles attenuates the turbulence and causes a decrease in the momentum spread.

Axial and lateral components of air turbulent intensities in the developed region of the jet are shown in Figs. 7 and 8, respectively. In the region $X / I)=14$ 18 the air turbulent intensities of the $w^{-{ }^{-}}$phase jet are $30-50 \%$ lower than those of the clean-air jet in the jet centerline region, where most of the particles exist. This means that the fluctuating motions of the jet are considerably affected by the presence of particles. even when $m$ is as small as 0.6 . The calculated radial turbulence intensity distributions of the two phase jet shown in Fig. 8 indicate clear concaves in the jet centerline region. An intensity distribution with a clear concave in the jet centerline region means that the turbulence in the jet is undeveloped. Therefore, these data also show the delay of turbulence development of the two-phase jet due to the presence of particles.

Figures 9 and 10 show the radial distributions of mean axial particle velocities $U_{p}$ in the initial region which includes the transitional region, and in the developed region, respectively. In Figs. 10-16, Hardalupas et al.'s experimental data ${ }^{(13)}$, of which the Stokes number is the same as that of the present investigation, are shown with our own experimental data. Their mass mixture ratio $m$ of gas and particles and nondimensional particle velocity $U_{p 0} / \delta_{0}$ at the nozzle outlet are $m=0.86$ and $U_{p_{0}} / U_{0}=0.86$.

As shown in Fig. 9, the calculated results in the initial region well represent the experimental data. Particles leaving the nozzle outlet with constant velocity are accelerated downstream and the velocity distributions of particles are gradually formed accord ing to air velocity distribution. The calculated results in the developed region of the jet also well represent the experimental data, including Hardalupas et al.'s data at $X / D=10$. There is a discrepancy between our calculated results and Hardalupas et al.'s experimental data at $X / D=20$. There are some differences in conditions between our simulation and their experiment, namely, our mass mixture ratio and our particle nozzle outlet velocity are $m=0.6$ and $U_{p_{0}} / U_{0}=0.43$, whereas their values are $m=0.86$ and $\zeta_{p_{0}} / U_{0}=0.86$. These differences would cause the discrepancy. The particle velocity decreases with increasing distance from the jet centerline. However, the velocity decreases are much smaller than those in air. Both the calculated and the experimental particle velocities at $\eta=0.1$ are $70-80 \%$ of the jet centerline particle 
velocity, while the air velocities at $\eta=0.1$ in the twophase jet are about $30 \%$ of the jet centerline air velocity, due to the particle inertia. In the outer region, $\eta(=Y / X)>0.1$, there are no data since the

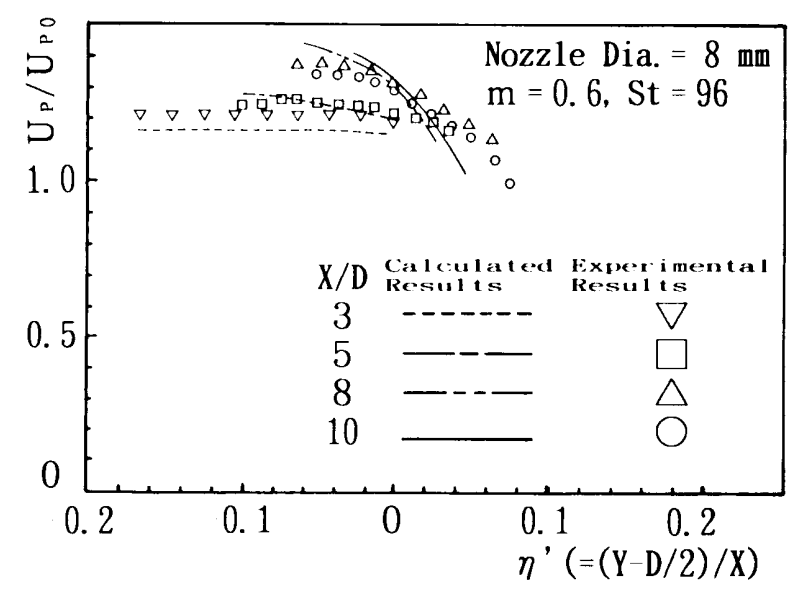

Fig. 9 Mean particle axial velocity distributions in initial and transitional regions of jets

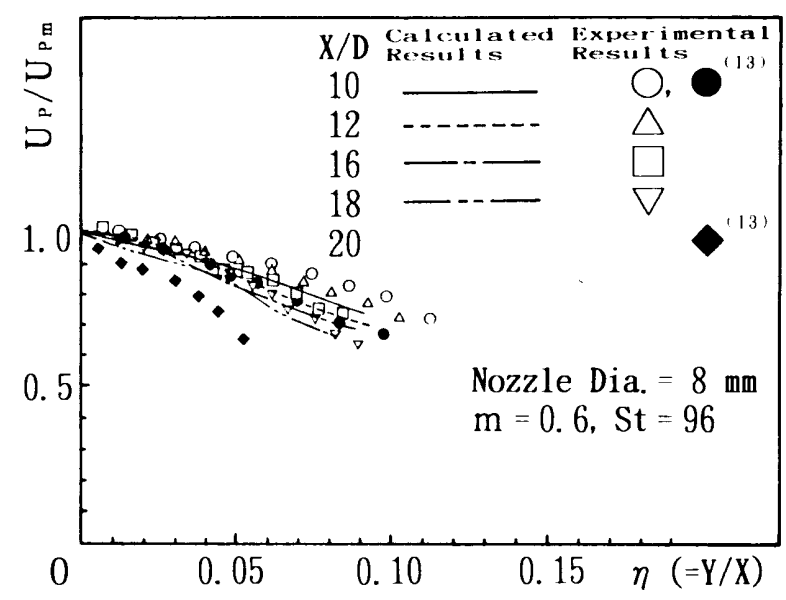

Fig. 10 Mean particle axial velocity distributions in transitional and developed regions of jets

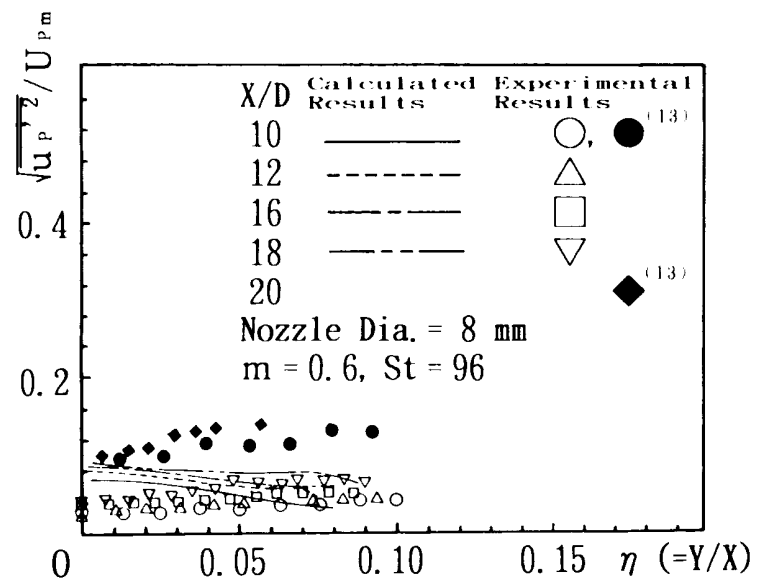

Fig. 11 Particle axial turbulent intensity distributions particle concentrations become so dilute that usable data cannot be obtained in this region.

Figure 11 shows intensity distributions of the particle axial fluctuating velocity $u_{p}^{\prime}$ normalized by $U_{p m}$. The difference between Hardalupas et al.'s data measured using the phase Doppler anemometer and our data measured using L. D. A. is considerable. The calculated results fall between the two sets of experimental data. Comparison of the particle and the air axial velocity intensities in the two phase jet, shown in Fig. 7, shows that the axial particle velocity intensities are about $50-60 \%$ of the air intensities in the jet centerline region and that the profiles are flatter than those of air intensity, due to inertia of the particles. Figure 12 shows intensity distributions of particle radial fluctuating velocity $v_{p}^{\prime}$ normalized by $U_{p m}$. The values of the calculated results are nearly equal to Hardalupas et al.'s experimental data. The radial particle velocity intensities are about one-third of the axial particle velocity intensities shown in Fig. 12. This is because, owing to large inertias, particles having no initial radial velocity component at the nozzle outlet are not disturbed to the radial direction in a jet flow of which the radial mean velocity is very small.

Figure 13 shows mean particle concentration distributions on the jet centerline, $C_{m}$, where $C_{0}$ is particle concentration at the nozzle outlet. There are some differences between our experimental results and Hardalupas et al.'s data which were measured using a phase Doppler anemometer. The calculated results fall between the two sets of experimental data. $C_{m} / C_{0}$ distributions are nearly the same as $U_{m} / U_{0}$ distributions.

Figures 14 and 15 show the radial mean particle concentration distributions in which the abscissas are $Y / D$ and $\eta$, respectively, where $C$ is mean particle concentration. The calculated distributions are slightly narrower than experimental ones. Figure 14 shows

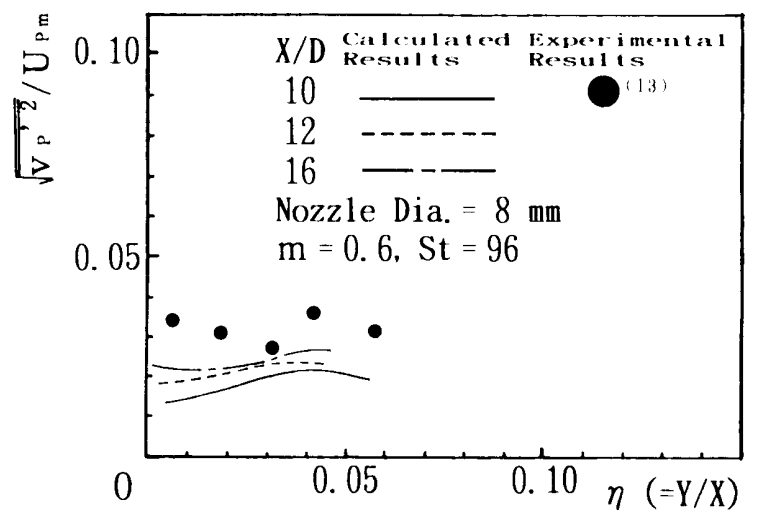

Fig. 12 Particle radial turbulent intensity distributions 


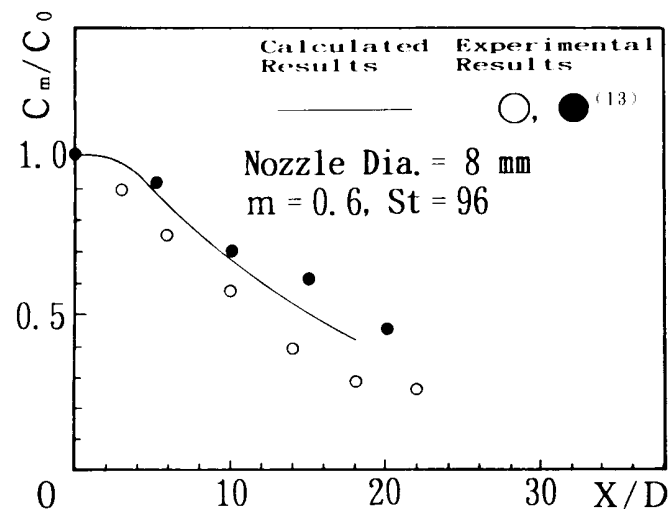

Fig. 13 Mean particle concentration distributions on the jet centerline

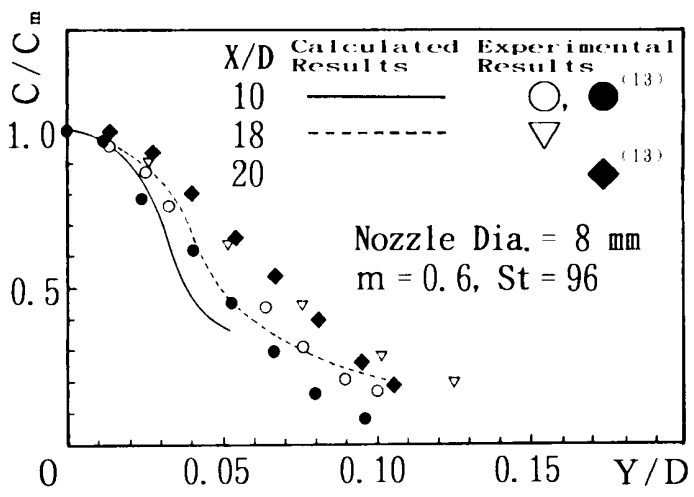

Fig. $14 \quad C / C_{m}$ is $Y / D$

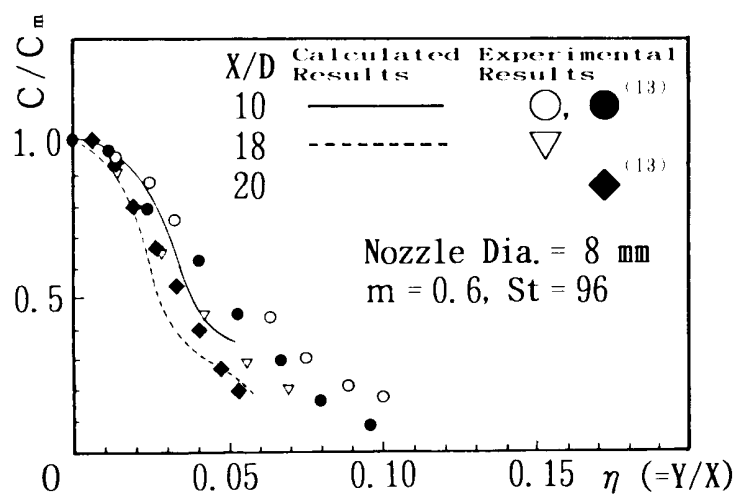

Fig. $15 C / C_{m}$ vs $\eta$

that the particles disperse to the outer region of the jet according to the air flow development downstream. Hardalupas et al. ${ }^{(13)}$ mentioned that the effect of the mass mixture ratio on the particle concentration distribution is small; accordingly our results are nearly equal to Hardalupas et al.'s experimental data. The comparison of Figs. 6 and 15 shows that the air velocity distributions are much wider than the particle concentration distributions in which there are few particles in the region $\eta>0.1$.

\section{Conclusion}

When one calculates medium Reynolds number turbulent two-phase flows using presently available supercomputers, the mesh sizes in computational domain are about seven times larger than the Kolmogorov microscale. This means that the calculated results do not describe eddies whose sizes are smaller than several times that of the eddy defined by the mesh size. However the calculated mean air and particle velocities, air and particle turbulence intensities and mean particle concentration are in fairly good agreement with experimental data. Hence the simulation well describes the motion of large ${ }^{-}$and medium-scale eddies which play an important role in the formation of turbulent gas-solid two-phase jet.

From the direct numerical simulation of the gassolid two-phase jet and the experiments, the following is clarified.

The decay of the particle velocity on the jet centerline is slower than that in air due to the large inertia of particles. In the upstream region, the air velocities of the two-phase jet are lower than those of the clean-air jet since the air is decelerated by the lower ${ }^{-}$speed particles. On the other hand air velocities of the two-phase jet in the downstream region are slightly higher than those of the clean-air jet since particles, whose velocities become higher than that of air, due to their inertias, draw air into the two- phase jet. The spread width of the two-phase jet is slightly narrower than that of the clean air jet in both the initial and the developed regions. This means that the presence of particles attenuates the turbulence and causes a decrease in the momentum spread. Due to the particle inertia, particle velocities at $\eta=0.1$ in the outer region of the jet are about $70 \%$ of the jet centerline particle velocity, while the air velocities at $\eta=0.1$ in the two ${ }^{-}$phase jet are about $30 \%$ of the jet centerline air velocity. The particle velocity inten. sities are smaller and the profiles are flatter than those of air in the two-phase jet, due to the particle inertias.

Particles, whose velocity and concentration are uniform at the nozzle outlet, become clustered downstream in the jet due to interactions with the large scale vortex motions. As the stream develops into turbulence, particles are gradually transported to the outer region of the jet. The simulation shows that there is a similar tendency for particle motion; the particles collect preferentially in regions of low vorticity and high strain rate in isotropic turbulence as reported by Squires and Eaton ${ }^{(8)}$, in the two- ${ }^{-}$phase jet.

\section{References}

(1) Yuu, S. and Umekage, T., Direct Numerical Simu- 
lation for Dilute Gas-Particle Jet, Gas-Solid Flows, ASME-FED, Vol. 121 (1991), p. 3.

(2) Squires, K. D. and Eaton, J. K., Particle Response and Turbulence Modification in Isotropic Turbulence, Phys. Fluids A, Vol. 2 (1990)), p. 1191.

(3) Schiller, L. and Naumann, A., ber die Grundlegenden Berechnungen bei der Schwerkraftaufbereitung, Z. Vereines Deutscher Inge., Vol. 77 (1933), p. 318.

(4) Grinstein, F. F., Oran, E. S. and Boris, J. P., Numerical Simulation of Asymmetric Mixing in Planar Shear Flows, J. Fluid Mech., Vol. 165 (1986), p. 201.

(5) Harlow, F. H. and Welch, J. E., Numerical Calculation of Time- Dependent Viscous Incompressible Flow of Fluid with Free Surface, Phys. Fluids, Vol. 8 (1965), p. 2182.

(6) Piacsek, S. A. and Williams. G. P., Conservation Properties of Convection Difference Schemes, J. Computational Physics, Vol. 6 (1970), p. 392.

(7) Laurence, J., Intensity, Scale and Spectra of Turbulence in Mixing Region of Free Subsonic
Jet, NACA Rept. 1292 (1956).

( 8 ) Squires, K. D. and Eaton, J. K., Preferential Concentration of Particles by Turbulence. Phys. Fluids A, Vol. 3 (1991), p. 1169.

(9) Chung, J. N. and Troutt, T. R., Simulation of Particle Dispersion in an Axisymmetric Jet, J. Fluid Mech., Vol. 186 (1988), p. 199.

(10) Townsend, A. A., The Structure of Turbulent Shear Flow, (1985), p. 184, Cambridge Univ. Press.

(11) Wygnanski, I. and Fiedler, H., Some Measurements in the Self- Preserving Jet, J. Fluid Mech., Vol. 38 (1969), p. 577.

(12) Panchapakesan, N. R. and Lumley, J. L., Turbulence Measurement in Axisymmetric Jets of Air and Helium. Part 1. Air Jet, J. Fluid Mech., Vol. 246 (1993), p. 197.

(13) Hardalupas, Y., Taylor, A.M.P.K. and Whitelow, J. H., Velocity and Particle-Flux Characteristics of Turbulent Particle-Laden Jets, Proc. Roy. Soc. London A, Vol. 426 (1989), p. 31. 